ARTÍCULO ORIGINAL

\title{
Manejo del derrame pericárdico en el paciente con cáncer
}

\author{
Luis Alberto Chinchilla-Trigos ${ }^{1}$, Edgardo Jiménez-Fuentes ${ }^{2}$, Abelardo Meneses-García ${ }^{3}$, \\ Mariana Cobos-Ortiz ${ }^{4}$
}

Palabras clave: neoplasias; pericardio; derrame pericárdico; técnicas de ventana pericárdica; supervivencia.

\begin{abstract}
Resumen
Introducción. El derrame pericárdico es la complicación cardiaca más frecuente en el paciente con cáncer. El cáncer de pulmón y el cáncer de mama son las neoplasias sólidas más frecuentemente asociadas con derrame pericárdico. El manejo oncológico multimodal ha permitido un aumento de la supervivencia global y ha expuesto complicaciones oncológicas que exigen manejo individualizado para estos pacientes.

Objetivo. Se describe la experiencia en el manejo del derrame pericárdico, desde su fisiopatología,
\end{abstract}

1 Cirujano oncólogo, Departamento de Oncología Torácica, Instituto Nacional de Cancerología, Ciudad de México, México; Universidad Nacional Autónoma de México, UNAM, Ciudad de México, México

2 Cirujano cardiotorácico; jefe, Departamento de Neumología y Cirugía de Tórax, Instituto Nacional de Cancerología, Ciudad de México, México; Universidad Nacional Autónoma de México, UNAM, Ciudad de México, México

3 Patólogo oncólogo; director general, Instituto Nacional de Cancerología, Ciudad de México, México; Universidad Nacional Autónoma de México, UNAM, Ciudad de México, México

4 Citopatóloga, Instituto Nacional de Cancerología, Ciudad de México, México; Universidad Nacional Autónoma de México, UNAM, Ciudad de México, México

Fecha de recibido: 2 de noviembre de 2016

Fecha de aprobación: 11 de abril de 2017

Citar como: Chinchilla-Trigos LA, Jiménez-Fuentes E, MenesesGarcía A, Cobos-Ortiz M. Manejo del derrame pericárdico en el paciente con cáncer. Rev Colomb Cir. 2017;32:82-93. la adecuada clasificación en derrame pericárdico asociado a neoplasia maligna, derrame pericárdico maligno o carcinomatosis pericárdica, hasta su abordaje diagnóstico y terapéutico.

Resultados. La incidencia del derrame pericárdico en nuestra institución es de $12 \%$. En casi 100 procedimientos en 11 años de ventana pericárdica, pericardiectomía e instalación de catéter subcutáneo (tunnelized) pericárdico o pleuropericárdico temporal, la mortalidad posoperatoria fue de 1,2\%, y la recurrencia del derrame pericárdico fue de 2,1\% comparada con una de $33 \%$ en los pacientes sometidos a pericardicentesis.

Conclusión. El derrame pericárdico maligno es una urgencia oncológica. Requiere manejo costo-efectivo en términos de ser resolutivo, expedito y duradero, sin agregar morbilidad en un paciente ya con deterioro de su estado general. La ventana pericárdica por toracoscopia (Video-Assisted Thoracoscopic Surgery, VATS) en pacientes seleccionados y la minitoracotomía antero-lateral son la vía ideal de abordaje del paciente con derrame pericárdico maligno.

\section{Introducción}

El derrame pericárdico es la complicación cardiaca más frecuente ( 6 a $10 \%$ ) en el paciente con cáncer. En una serie de autopsias de pacientes oncológicos, el 8,1 \% tenía derrame pericárdico; de estos, el 2,7\% había presentado, al menos, un signo de derrame pericárdico ${ }^{1}$.

El cáncer puede afectar el pericardio por cuatro mecanismos: 
1. implante de células neoplásicas en el pericardio por extensión directa o por diseminación hematógena o linfática, desde el tumor primario;

2. toxicidad de la quimioterapia;

3. toxicidad de la radioterapia, o

4. infecciones oportunistas, en relación con la inmunosupresión por agentes citotóxicos y la inmunoterapia $^{2}$.

Antes del advenimiento del manejo multimodal (quimioterapia, radioterapia y terapias blanco), la incidencia del derrame pericárdico era del $21 \%$, con una supervivencia global menor de12 semanas después del drenaje y una recurrencia hasta de $40 \%$ del derrame pericárdico. Actualmente, en los centros de referencia, el derrame pericárdico maligno sigue siendo la complicación cardiaca más frecuente, aunque con una incidencia menor de 1 a $10 \%$. Con la adecuada evacuación del derrame pericárdico, la supervivencia global aumenta a 28 semanas y puede ser superior, hasta 20 años, cuando no hay recurrencia del derrame pericárdico y según los factores pronósticos del paciente ${ }^{3,4}$.

Con la descripción del saco pericárdico, surgieron hipótesis sobre la acumulación de líquido en su espacio, que comprimiría y entorpecería la mecánica de bomba del corazón. En 1829, Dominique Jean Larrey describió la ventana pericárdica como una comunicación libre y persistente entre el pericardio y la cavidad pleural, los tejidos subcutáneos y el medio externo. La pericardiocentesis, drenaje percutáneo del saco pericárdico, fue descrita solo hasta 1840. Casi 200 años después, se continúa debatiendo sobre cuál es la mejor manera de paliar el derrame pericárdico en el paciente con cáncer, considerando la expectativa de vida y la posibilidad de continuar el manejo oncológico del tumor primario ${ }^{5}$.

El cáncer de pulmón representa el 12,9\% de todas las neoplasias malignas, ocupa el primer lugar de incidencia en hombres y el tercer lugar en mujeres después del cáncer de mama y colorrectal, y es la principal causa de muerte por cáncer a nivel mundial ${ }^{1,6}$. El cáncer de pulmón es el tumor primario que más frecuentemente compromete el pericardio. El cáncer de mama es el tumor maligno más frecuente y prevalente en la mujer en los países desarrollados. El avance en el manejo multimodal de estos dos tipos de cáncer ha permitido el aumento de la supervivencia global en cada una de sus etapas clínicas y ha expuesto complicaciones oncológicas que exigen el manejo individualizado, teniendo en cuenta el estado general del paciente, la expectativa de vida y la posibilidad de recibir el tratamiento idóneo para el tumor primario ${ }^{7-9}$.

\section{Historia natural del derrame pericárdico en el paciente con cáncer}

El entendimiento de la disposición del saco pericárdico es fundamental. El pericardio tiene dos hojas. La externa (pericardio fibroso) es más gruesa, con fibras de colágeno y elastina que, principalmente, proporcionan elasticidad. La interna (pericardio seroso) es delgada y transparente; se subdivide en una capa serosa parietal, la cual está fusionada con el pericardio fibroso, y una capa serosa visceral, la cual está en íntimo contacto con el epicardio y la adventicia de los grandes vasos. El espacio pericárdico, entre el pericardio fibroso y el seroso, contiene menos de $50 \mathrm{ml}$ de líquido pericárdico, que es un ultrafiltrado de plasma. La fisiopatología del derrame pericárdico en el paciente con cáncer debe asumirse como una urgencia oncológica, diferente al derrame pericárdico asociado a trauma, falla cardiaca $\mathrm{u}$ otras enfermedades debilitantes ${ }^{10-12}$.

La evaluación y el manejo del derrame pericárdico exigen tener claridad en las siguientes definiciones.

- Derrame pericárdico asociado a neoplasia maligna: acumulación de líquido en el saco pericárdico en el paciente oncológico, sin células malignas en el examen citológico o el bloque celular del líquido evacuado (caso clínico 1).

- Derrame pericárdico maligno: acumulación de líquido en el saco pericárdico con células neoplásicas malignas en el examen citológico o el bloque celular, sin células neoplásicas en el pericardio (caso clínico 2).

- Carcinomatosis pericárdica: identificación macroscópica o microscópica de implantes de células malignas en las hojas del saco pericárdico, con células neoplásicas en el líquido pericárdico o sin ellas (caso clínico 3). 
Entre las causas de derrame pericárdico asociado a neoplasia maligna, se encuentra la quimiotoxicidad con medicamentos como ciclofosfamida, citarabina, doxorrubicina o gencitabina, reconocidos por causar insuficiencia cardiaca, miocarditis y pericarditis, hasta en $20 \%$ de los pacientes cuyos esquemas de quimioterapia se basan en estos medicamentos ${ }^{13}$. Los campos amplios de radioterapia en el tórax, como aquella en manto para el linfoma, la de ciclo mamario completo o la posterior a mastectomía, lesionan el cardiomiocito y alteran la mecánica valvular, lo cual favorece la insuficiencia cardiaca, el espasmo coronario y la fibrosis del pericardio.

En los pacientes con compromiso inmunológico por terapia sistémica citotóxica o terapias blanco, como ocurre con los inhibidores de la tirosín-cinasa (Tyrosine Kinase Inhibitor, TKI) contra el receptor para el factor de crecimiento epidérmico (Epidermal Growth Factor Receptor, EGFR) como afatinib o erlotinib, entre otros usados en el cáncer de pulmón de células no pequeñas, se presentan infecciones oportunistas por citomegalovirus, micobacterias y hongos como Aspergillus spp. y Candida spp.; también, sucede así con el bevacizumab, anticuerpo monoclonal contra el factor de crecimiento del endotelio vascular (Vascular Endothelial Growth Factor, VEGF) utilizado en cáncer de mama, con inmunomoduladores como el nivolumab, y con inhibidores de la vía PD1- PDL-1 en el cáncer de pulmón y el mesotelioma pleural maligno ${ }^{2,14}$.

Por otra parte, se presenta acumulación lenta pero progresiva de líquido pericárdico por alteración de la superficie del pericardio. La obstrucción del flujo linfático normal en el mediastino por la actividad tumoral, ocurre en tumores del mediastino, linfoma y tumores germinales. La siembra de células malignas en el pericardio se inicia en la superficie del epicardio, continúa en el pericardio visceral y, finalmente, en el pericardio parietal, cuando la vía de diseminación del tumor primario es hematógena y linfática, como ocurre en el adenocarcinoma de pulmón y el de mama, entre otros ${ }^{15}$.

En la diseminación por contigüidad al pericardio por vía transcelómica, el depósito de células neoplásicas ocurre desde el pericardio parietal y posteriormente al visceral, como es usual en el cáncer de pulmón de células pequeñas, el mesotelioma maligno pleural, el adenocarcinoma de ovario y los subtipos de tumores de células en anillo de sello. La alteración de la superficie mesotelial de las hojas del pericardio, además de trastornar la secreción y la absorción del líquido pericárdico, permite el desarrollo de cicatrices y adherencias entre el pericardio fibroso y el visceral, lo que genera tabiques que impiden el libre flujo del líquido pericárdico y, por ende, su función amortiguadora de la variación respiratoria que se transmite a las cámaras cardiacas.

Este último punto se reconoce como una de la causas de recurrencia del derrame pericárdico después de la pericardiocentesis, pues los tabiques no permiten la evacuación de todo el líquido pericárdico acumulado ${ }^{16}$. La ventana pericárdica, la pericardiectomía y la instalación de un sistema temporal de drenaje pericárdico establecen un campo de maniobra para la resección amplia de la hoja del pericardio parietal, así como para la identificación de implantes macroscópicos y la toma de tejido para estudio histopatológico ${ }^{17,18}$.

\section{Características clínicas y factores de pronóstico}

La presencia de derrame pericárdico reduce la posibilidad de cualquier tipo de tratamiento paliativo diferente a las medidas de mejor soporte médico, a menos de $2 \%$ de los pacientes con esta complicación ${ }^{19}$.

El espectro clínico del paciente oncológico con derrame pericárdico incluye:

1. derrame pericárdico sin repercusión hemodinámica,

2. derrame pericárdico con algún signo de repercusión hemodinámica, $\mathrm{y}$

3. taponamiento cardiaco.

Todo lo anterior se relaciona con la velocidad de acumulación de líquido en el saco pericárdico, la rigidez y la variación de la superficie mesotelial de las hojas del pericardio ${ }^{17-19}$.

En cerca de $70 \%$ de los casos, el derrame pericárdico en el paciente con cáncer es un hallazgo incidental durante la evaluación diagnóstica, el tratamiento o el seguimiento del tumor primario. En $60 \%$ se presenta, al menos, un signo de repercusión hemodinámica, el cual tampoco refleja la gravedad o intensidad del derrame pericárdico, dado que $50 \%$ de los casos se acompañan 
de derrame pleural, edema pulmonar, desnutrición y cardiotoxicidad por la terapia para el tumor primario.

En el $80 \%$ de los casos, el signo de repercusión hemodinámica más frecuente es la taquicardia. La disnea, la taquipnea y el pulso paradójico se encuentran en 60,45 y $40 \%$, respectivamente; la tos, la ortopnea y la hipotensión se presentan en menos del $25 \%$ de los casos y son signos inminentes de taponamiento cardiaco.

Finalmente, la clásica tríada de Beck, taquicardia, hipotensión y disminución de los ruidos cardiacos, se encuentra en menos del $15 \%$ de los derrame pericárdicos malignos.

Se han descrito múltiples opciones de tratamiento del derrame pericárdico en el paciente con cáncer, con base en la repercusión hemodinámica, los factores de pronóstico, como el estado funcional evaluado mediante el índice de Karnofsky y la escala ECOG (Eastern Cooperative Oncology Group), la expectativa de vida, la histopatología y el comportamiento biológico del tumor, y también, los factores predictores de reacción al tratamiento oncológico ${ }^{20,21}$.

\section{Principios del tratamiento del derrame pericárdico en pacientes oncológicos}

El manejo ideal del derrame pericárdico en el paciente con cáncer, debe ser una medida costo-efectiva, resolutiva, expedita y duradera, teniendo presente la menor morbilidad agregada, la expectativa de vida y la recurrencia del derrame pericárdico.

Las opciones quirúrgicas tradicionales, como la ventana pericárdica por vía subxifoidea, por toracotomía antero-lateral y postero-lateral o por toracoscopia, la aplicación de agentes que producen esclerosis en el pericardio, la instalación temporal de un catéter de drenaje en el saco pericárdico y la pericardiocentesis guiada por imágenes, requieren de un agudo criterio oncológico, habilidad quirúrgica y conocimiento de la fisiología cardiopulmonar ${ }^{22-24}$.

Todos estos procedimientos son bien tolerados por el paciente que se encuentra fuera del control médico quirúrgico de su neoplasia primaria. No obstante, se ha encontrado disminución de la recurrencia del derrame pericárdico (15\%) y mayor supervivencia global a los siete meses, en los pacientes sometidos a ventana pericárdica por toracotomía antero-lateral izquierda, frente a $31 \%$ de recurrencia y supervivencia a solo tres meses en el grupo con drenaje percutáneo guiado por imágenes ${ }^{25}$.

En una serie de 1.841 pericardiocentesis, se encontró que la supervivencia del paciente se relacionaba con el tipo histológico del tumor primario y la posibilidad de tratamiento sistémico, con supervivencia mayor de tres meses para el cáncer de mama del $93 \% \mathrm{y}$ del $72 \%{ }^{9}$.

En el trabajo de Patel, et al., con 1.399 pacientes, se comparó el tratamiento percutáneo definitivo con el procedimiento abierto más la inserción de un catéter temporal, portátil, de drenaje portable, se demostró igual morbilidad: $6 \%$ en el grupo de pericardiocentesis y $8 \%$ con la técnica abierta.

En 14 pacientes se presentó infección del catéter temporal subcutáneo (tunnelized) pericárdico ('tunelitis'), la cual no fue grave y se asoció con un mal cuidado del mismo; fue necesario retirar el catéter dentro de los 20 días después de su instalación ${ }^{26}$.

En la serie del Memorial Sloan Kettering, se evaluaron 72 pacientes con derrame pericárdico, 37 sometidos a pericardiocentesis guiada por imágenes más la aplicación de un agente esclerosante intrapericárdico y, 35 pacientes, a ventana pericárdica quirúrgica. La supervivencia en ambos grupos fue de 97 días y el costo fue mayor en el grupo quirúrgico. La recurrencia del derrame fue igual con el drenaje percutáneo y la ventana pericárdica quirúrgica, gracias a la instilación del agente esclerosante ${ }^{27}$.Con la instilación de un agente citotóxico intrapericárdico, como el cisplatino, el resultado ha sido menos alentador, pues no aumentó la supervivencia global, pero sí se presentó toxicidad hematológica ${ }^{28,29}$.

\section{Caso clínico 1. Derrame pericárdico asociado a neoplasia maligna}

Se trata de una mujer de 38 años con cáncer bilateral de mama, sincrónico, triple negativo y localmente avanzado, que recibió quimioterapia neoadyuvante y, posteriormente, se sometió a mastectomía radical bilateral modificada de tipo Madden, y radioterapia posoperatoria. La reconstrucción mamaria fue tardía y por etapas. 
Tuvo un periodo de dos años libre de enfermedad $\mathrm{y}$, durante su vigilancia y seguimiento, refirió disnea progresiva de medianos esfuerzos y de un mes de evolución. El índice de Karnofsky fue de 70 \% y presentó taquicardia sinusal.

En el electrocardiograma se encontraron signos de bajo voltaje y ausencia de la onda $p$. En la radiografía de tórax se observó ensanchamiento de la silueta cardiomediastínica y borramiento del ángulo costofrénico izquierdo (figura 1). En la tomografía de tórax (TC), se observó un derrame pericárdico con separación de 3,5 $\mathrm{cm}$ entre las hojas del pericardio y un derrame pleural izquierdo (figura 2).

Se realizó una ventana pericárdica más pericardiectomía e instalación de un catéter pleuro-pericárdico subcutáneo (tunnelized) mediante toracoscopia izquierda. El líquido pericárdico era de color cetrino. No se identificó carcinomatosis pleural o pericárdica. El examen citológico del líquido pericárdico fue negativo para neoplasia maligna y en la histopatología de las biopsias de pericardio y de pleura parietal se observó un mesotelio reactivo, negativo paraneoplasia maligna (figura 3 ).

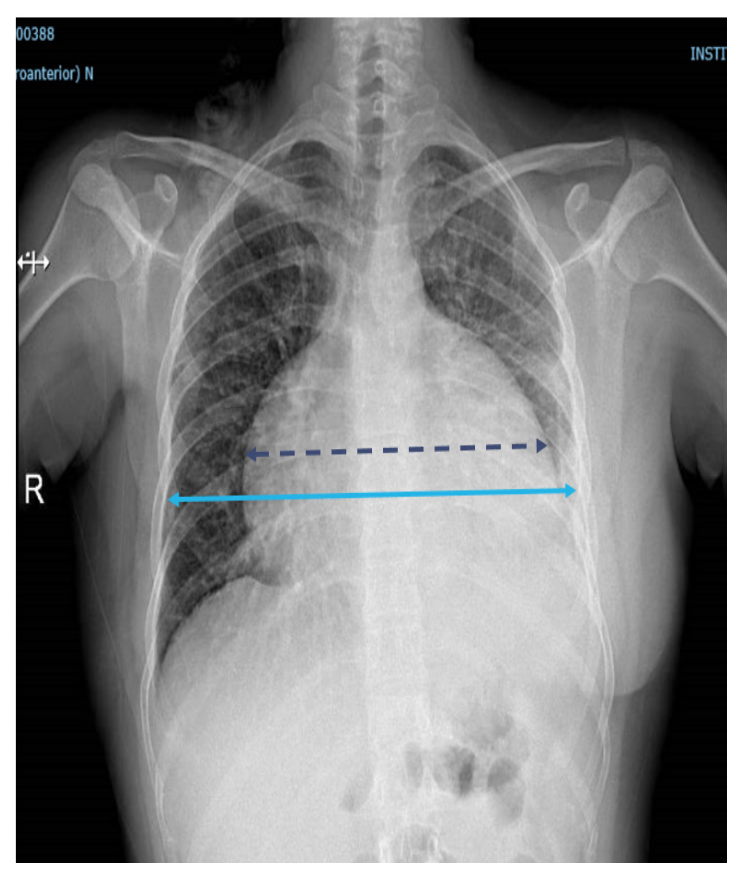

FIgURA 1. Radiografía de tórax: derrame pericárdico, ensanchamiento de la silueta cardiomediastínica (línea punteada) y derrame pleural izquierdo

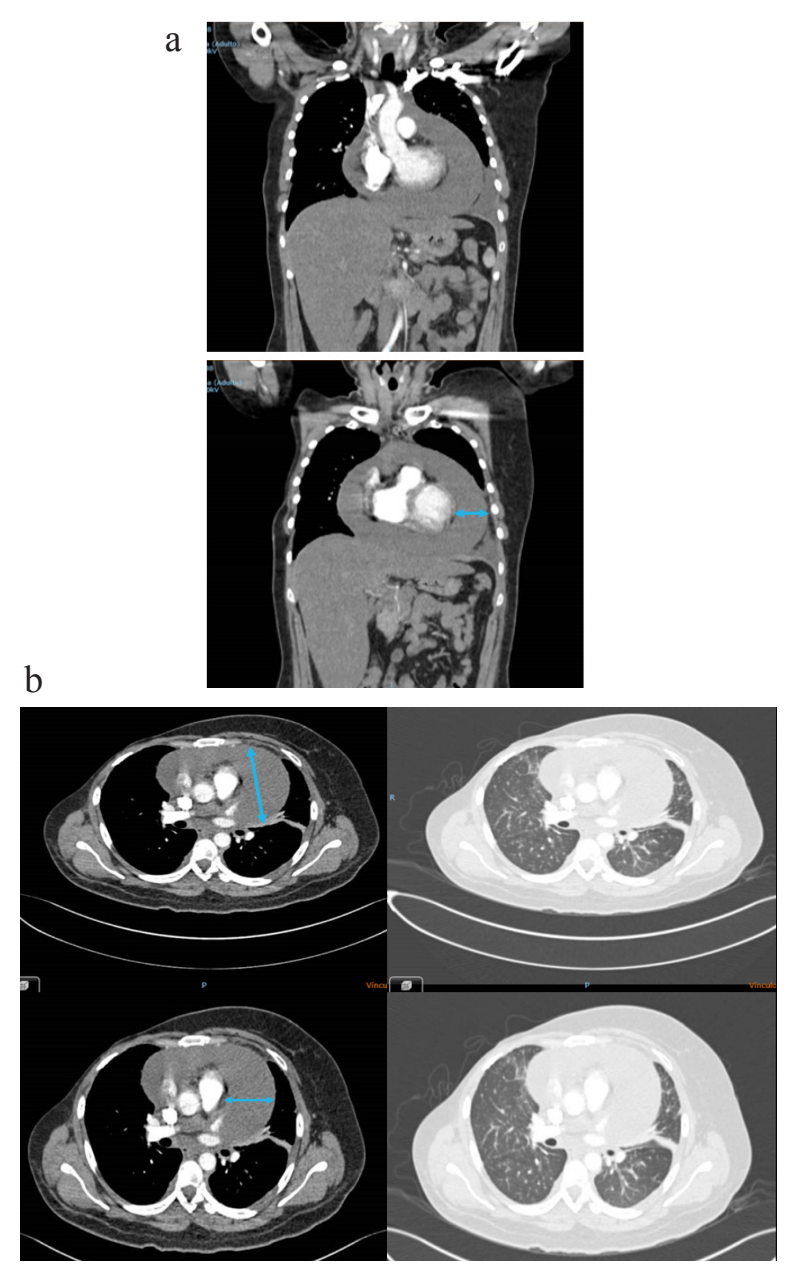

Figura 2. TC de tórax. a. Cortes coronales: derrame pericárdico homogéneo y sin tabiques. b. Cortes axiales: separación de 3,5 cm entre las hojas del pericardio (doble flecha). No se observa engrosamiento del pericardio y hay un escaso derrame pleural izquierdo.

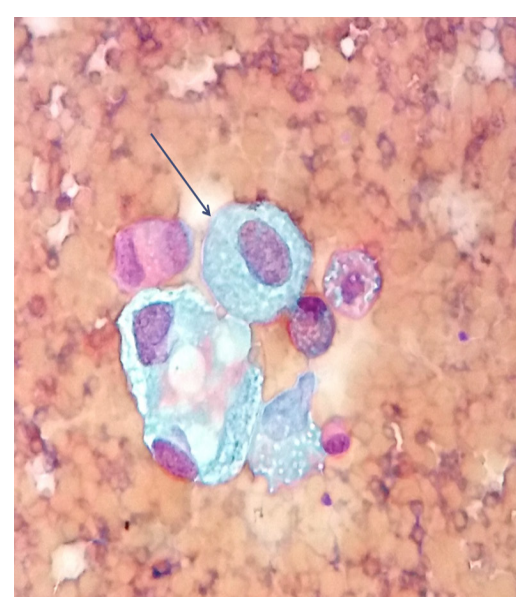

Figura 3. Citología de líquido pericárdico. Se observa un fondo hemorrágico, células mesoteliales con cambios reactivos y discreto aumento del núcleo y el citoplasma (flecha). Diagnóstico: mesotelio reactivo. Tinción de Papanicolaou, $40 X$. 
El drenaje pleuro-pericárdico temporal se mantuvo durante tres semanas hasta que los gastos fueron menores de $50 \mathrm{ml}$. La radiografía de tórax mostró la posición del catéter pleuropericárdico, una adecuada expansión pulmonar izquierda y disminución del tamaño de la silueta cardiomediastínica (figura 4). La paciente recibió rehabilitación cardiopulmonar (figura 5).

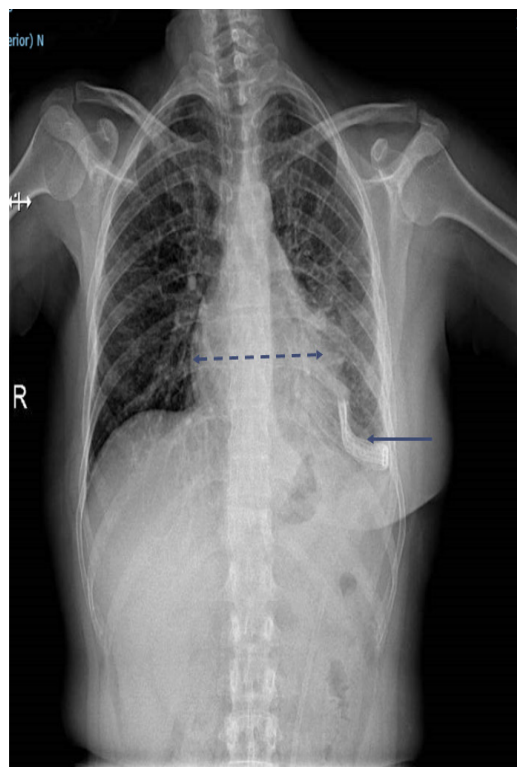

Figura 4. Radiografía de tórax después de tres semanas de la ventana pericárdica: catéter pleuropericárdico izquierdo (flecha), adecuada expansión pulmonar izquierda y disminución del tamaño de la silueta cardiomediastínica
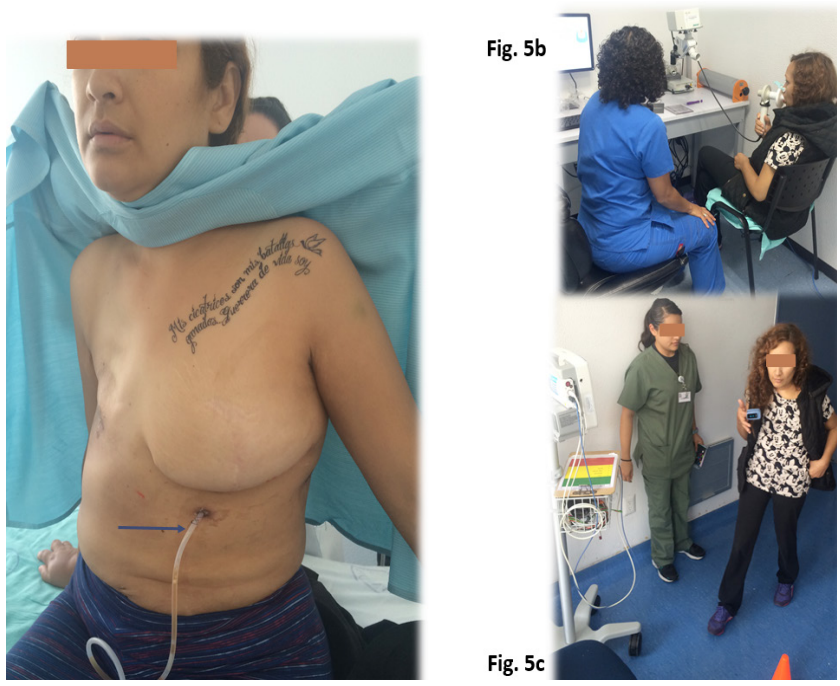

Figura 5. a. Catéter pleuropericárdico (flecha) permeable y sin signos de infección después de tres semanas de instalado. b y c. Rehabilitación cardiopulmonar posoperatoria
En la evaluación ecocardiográfica de la función ventricular, la fracción de eyección fue de 55 \% y hubo signos de insuficiencia cardiaca asociados a cardiotoxicidad.

\section{Caso clínico 2. Derrame pericárdico maligno}

Se trata de una mujer de 67 años con adenocarcinoma de pulmón $\mathrm{T}_{3} \mathrm{~N}_{2} \mathrm{M}_{1 \mathrm{a}}$, en etapa clínica IV y 2 en la escala ECOG.

Después de la quimioterapia, en la tomografía por emisión de positrones (PET-CT) se observaron los implantes pleurales, con engrosamiento pleural y pericárdico, y derrame pericárdico. Hubo progresión según los criterios de reacción por PET-CT (Positron Emission Tomography Response Criteria, PERCIST). No se observaron signos de taponamiento cardiaco (figura 6).

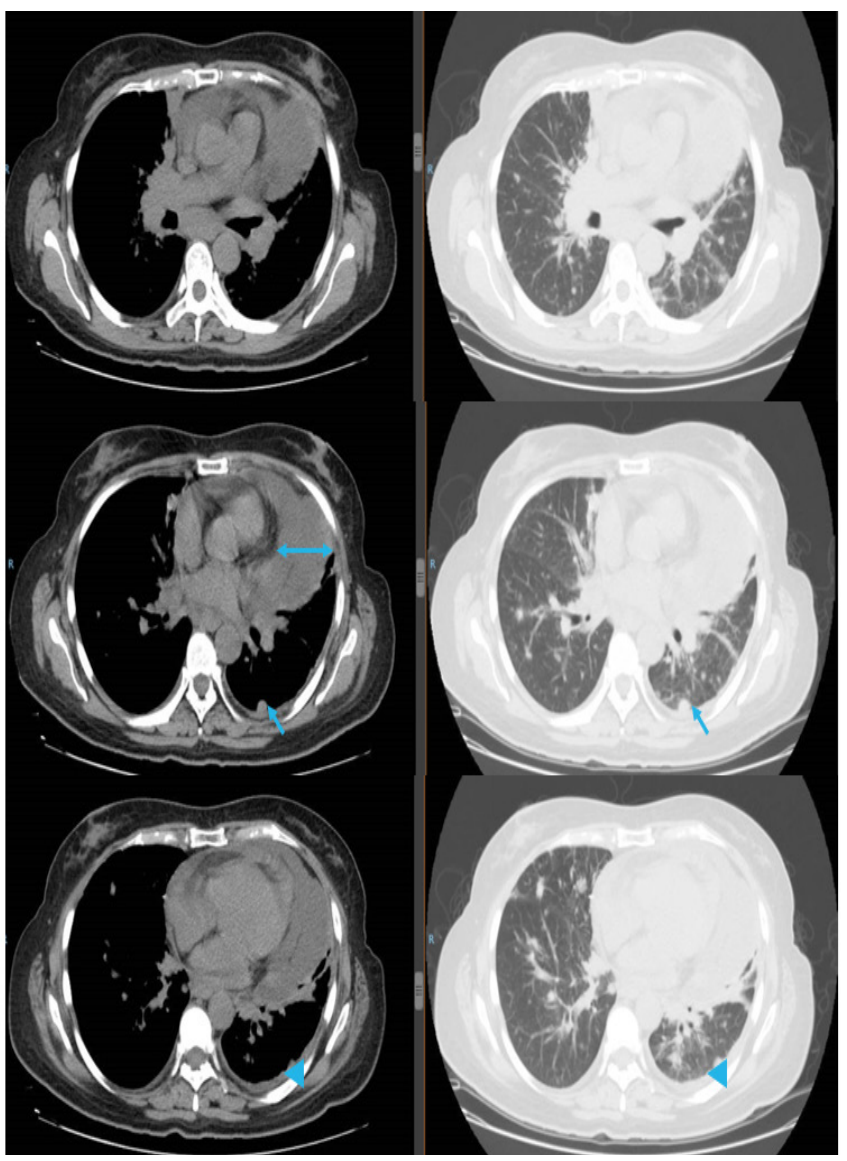

FIguRA 6. PEC-CT, ventanas mediastinal y pulmonar: derrame pericárdico. La doble flecha muestra una separación de $2 \mathrm{~cm}$ del pericardio, la flecha señala los implantes pleurales y, la cabeza de flecha, el engrosamiento pleural. 
Se practicó una ventana pericárdica subxifoidea con instalación de un catéter subcutáneo (tunnelized) pericárdico (figura 7).

El estudio citológico del líquido pericárdico y del bloque celular mostró características serohemáticas; fue positivo para neoplasia maligna: carcinoma (figura 8). La histopatología del tejido obtenido de la ventana pericárdica fue negativa para neoplasia maligna.

Seis semanas después, la paciente tuvo que ser sometida a toracoscopia para evacuar el derrame pleural izquierdo; se tomaron biopsias pleural y pulmonar para evaluar el perfil molecular en las muestras de tejido.
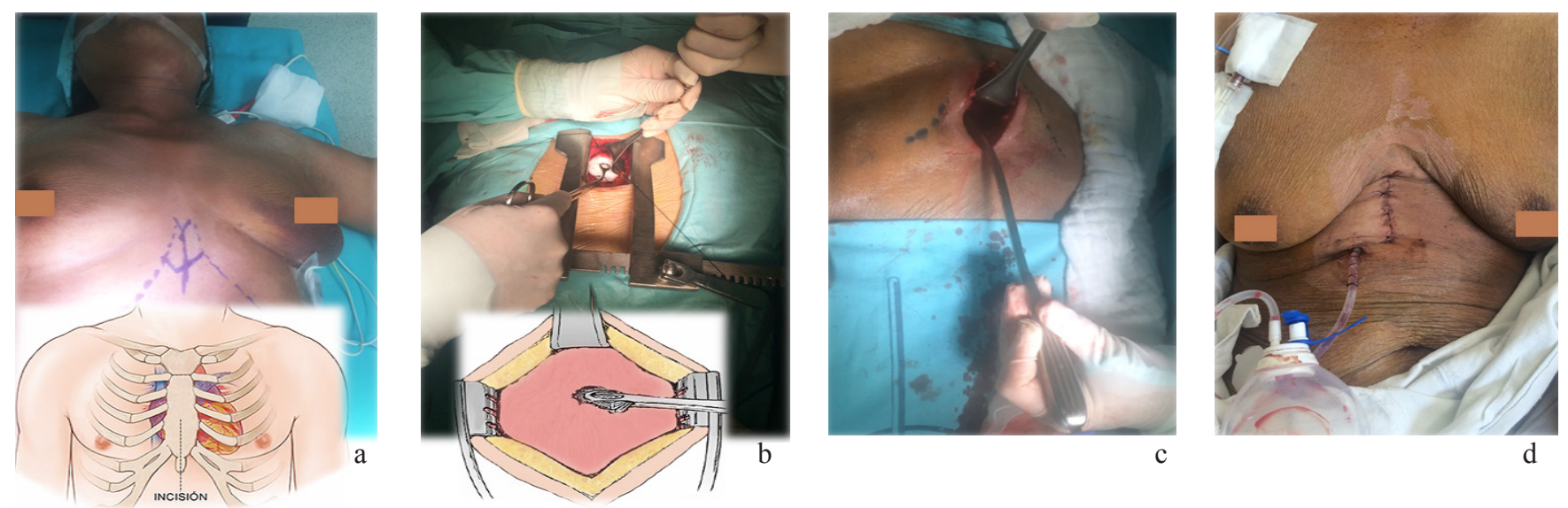

FIGURA 7. Ventana pericárdica subxifoidea e instalación del catéter pericárdico subcutáneo (tunnelized): a) Marcación del apéndice xifoides y límites de la incisión; b) Exposición del saco pericárdico con separador de Finochietto, disección roma y separación del diafragma y la grasa mediastinal; c) Evacuación del derrame pericárdico; d) Instalación del catéter subcutáneo (tunnelized) pericárdico
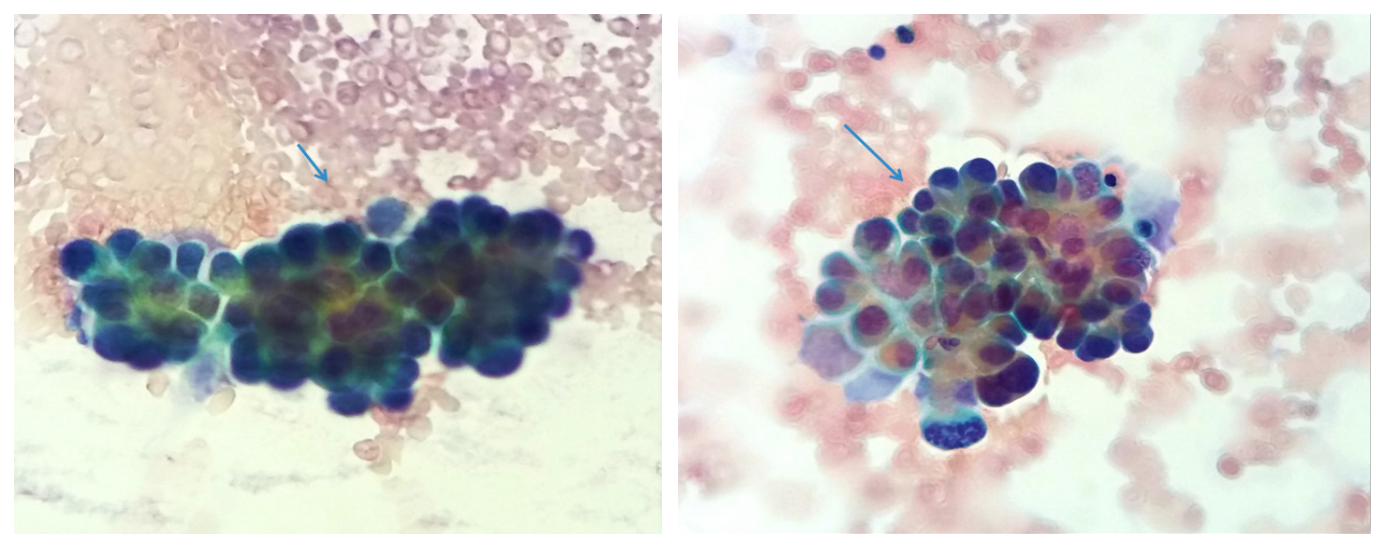

FIGURA 8. Estudio citológico del derrame pericárdico maligno: se observa un fondo hemorrágico (flecha corta) con abundantes células neoplásicas que forman mórulas y rosetas (flecha larga). Tienen citoplasma amplio y núcleos grandes y pleomorfos. Diagnóstico citológico de líquido pericárdico: carcinoma. Tinción de Papanicolaou, $40 X$. 
cardiomediastínica (figura 9).En la TC de tórax se apreciaba derrame pericárdico, con tabiques, derrame pleural izquierdo, y engrosamiento pleural y pericárdico (figura 10).

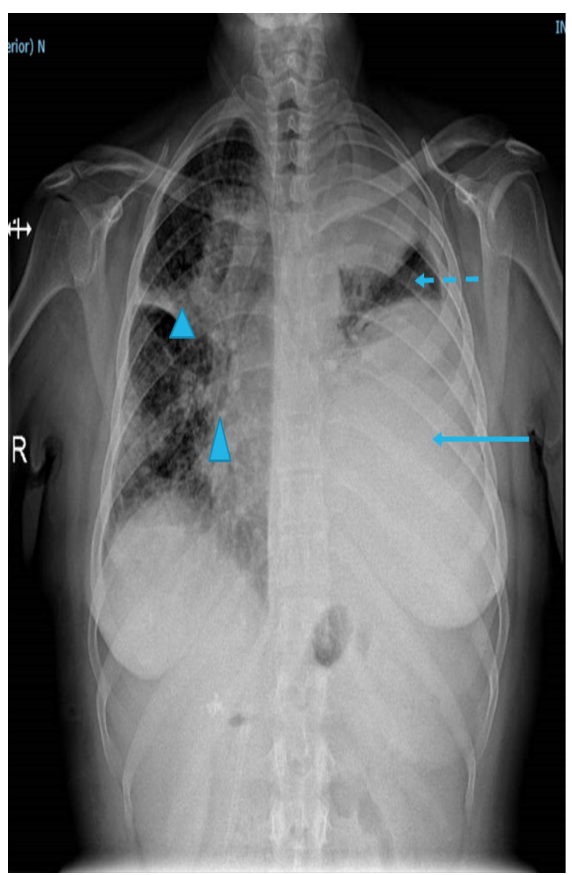

Figura 9. Radiografía de tórax: derrame pleural izquierdo. La flecha muestra opacidad de los dos tercios inferiores del hemitórax izquierdo y, la flecha punteada, 'atrapamiento' pulmonar'. En el hemitórax derecho, las opacidades (cabezas de flechas) sugieren linfangitis carcinomatosa, en el contexto de la enfermedad.
Se le practicó una ventana pericárdica izquierda mediante toracotomía asistida por video (VATS) y se le instaló un catéter subcutáneo (tunnelized) pleuropericárdico temporal (figura 11).

En el estudio citológico y del bloque celular del líquido pericárdico, se reportó neoplasia maligna: carcinoma. En la histopatología del pericardio se informó adenocarcinoma de mama. En la inmunohistoquímica, los receptores de estrógeno y de progesterona, la proteína GATA y la mamoglobina, fueron positivos, y el protooncogén HER2/neu, negativo (figura 12).

\section{Discusión}

Consideramos que, además de los factores conocidos involucrados en el desarrollo del derrame pericárdico, como la relación entre el volumen de líquido pericárdico y el tiempo de acumulación, en el caso del paciente oncológico, se suma el estado de la superficie de las hojas del saco pericardio. Por esta razón, los pacientes con derrame pericárdico asociado a neoplasia maligna albergan volúmenes cuantiosos, entre 500 y $1.000 \mathrm{ml}$, con sutiles cambios hemodinámicos. La formación de coágulos y tabiques favorece la recurrencia del derrame después de su evacuación.

Finalmente, en la carcinomatosis pericárdica, el pericardio fibroso y restrictivo causa que volúmenes
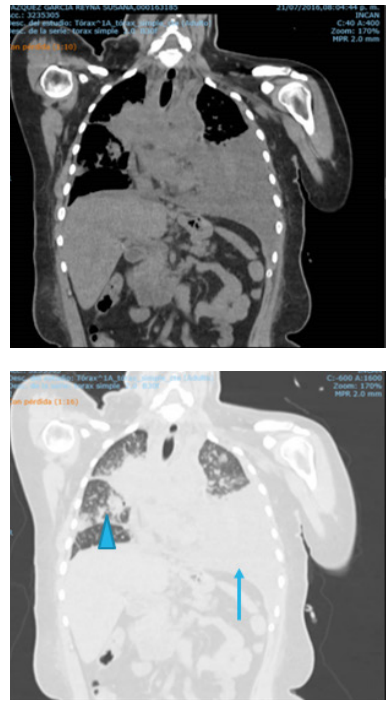

a

FiguRa 10. Tomografía axial de tórax: derrame pleural y pericárdico, y atrapamiento pulmonar; a) cortes coronales y b) cortes axiales. Las cabezas de flecha muestran imagen de linfangitis carcinomatosa, la flecha, el derrame pleural izquierdo y, la doble flecha, el derrame pericárdico con separación de 1,2 cm del pericardio. La flecha punteada indica las zonas de 'atrapamiento' pulmonar.

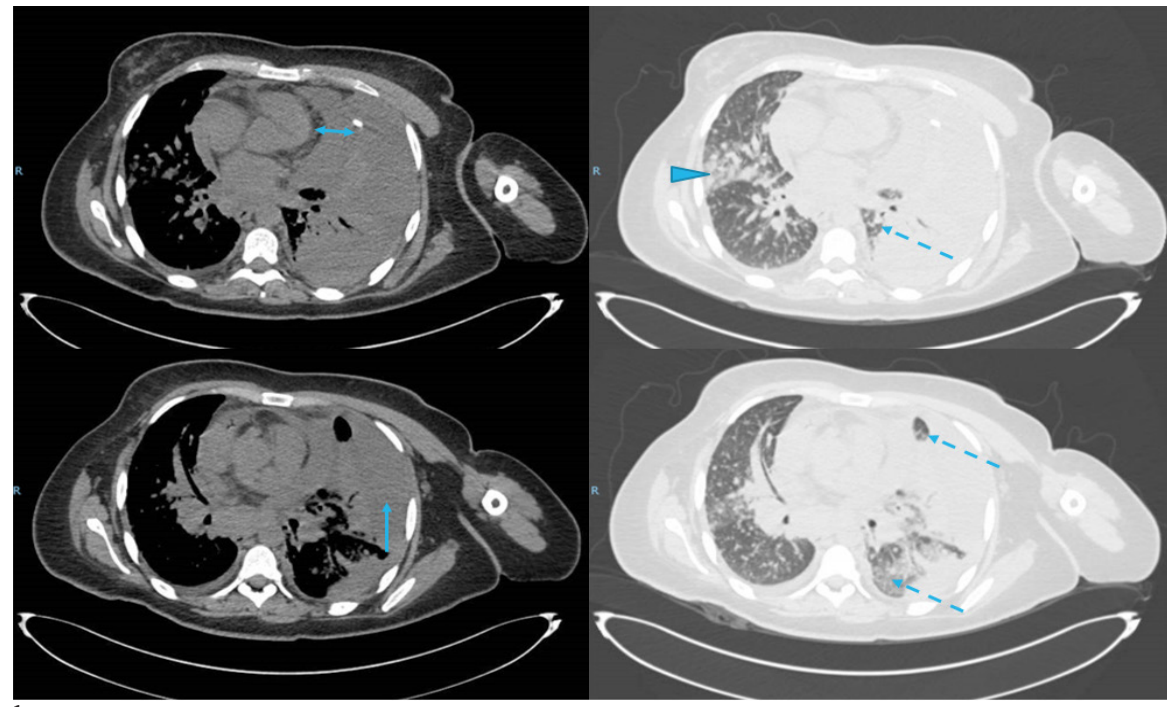

b 

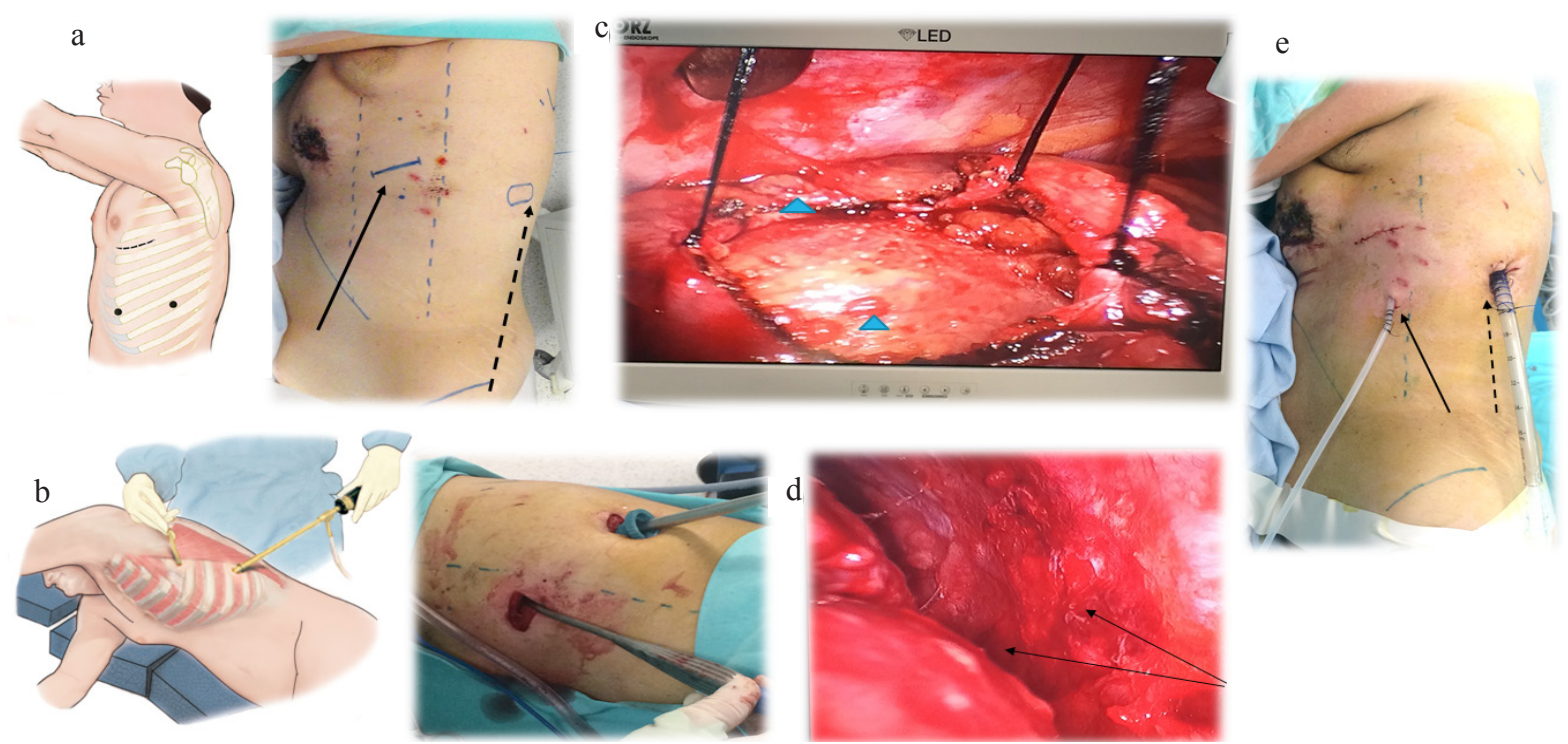

FigURA 11. Ventana pericárdica por VATS. a y b) El esquema y la fotografía muestran la disposición del paciente en decúbito lateral derecho. La línea punteada señala la incisión para el puerto de cámara en la evaluación toracoscópica. La flecha señala la incisión de trabajo de la VATS. c) Imagen del monitor de toracoscopia: las cabezas de flechas muestran los implantes tumorales en el pericardio parietal y el visceral, durante el procedimiento de la ventana pericárdica. d) Las flechas señalan implantes en la pleura visceral y la pulmonar. e) La flecha punteada muestra la utilización del puerto de cámara para colocar el tubo de tórax. La flecha muestra la salida del catéter subcutáneo (tunnelized) pleuropericárdico, dos a tres cm por debajo de la incisión, sobre la línea axilar anterior.

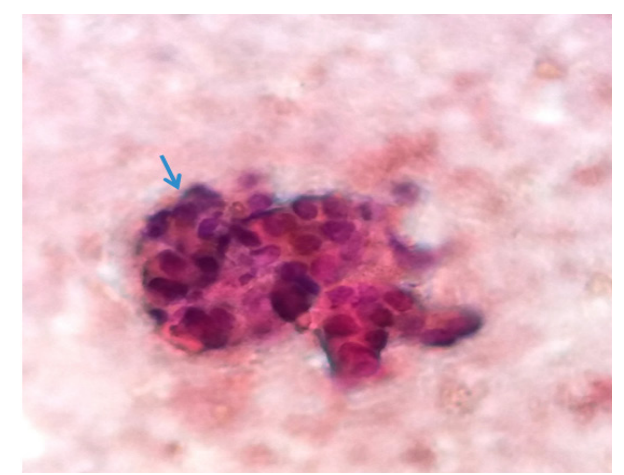

a

FIGURA 12.a) Citología de líquido pericárdico: se observa fondo hemorrágico con escasos grupos de células de aspecto epitelial (flecha) con ligera atipia. Tinción de Papanicolaou, 40X. b) Bloque celular de líquido pericárdico: se observan grupos de células epiteliales formando estructuras glandulares (flecha punteada) con núcleos ovales con ligera atípica, cromatina grumosa y escaso citoplasma; también, se observan escasas células mesoteliales. Hematoxilina y eosina, 100X.

pequeños de líquido conduzcan al taponamiento cardiaco. Usualmente, el ecocardiograma se considera el estudio estándar para determinar la presencia, la localización, el tamaño y la repercusión cardiaca del derrame pericárdico. La TC de tórax no es un buen método para evaluar el derrame pericárdico; sin embargo, la TC y la resonancia magnética pueden ser superiores al ecocardiograma para determinar la presencia y la

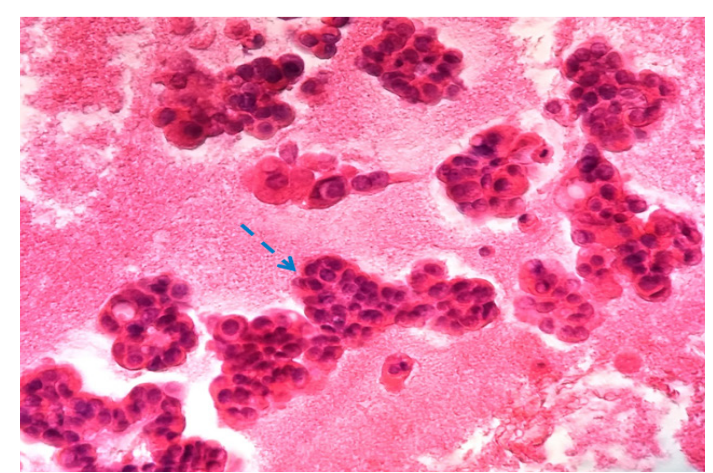

b 
con pericardiocentesis presenta una recurrencia del $33 \%{ }^{32}$.

Durante los últimos 11 años, en 96 procedimientos quirúrgicos de ventana pericárdica, pericardiectomía e instalación de catéter subcutáneo (tunnelized) pericárdico, practicados en pacientes con cáncer y derrame pericárdico, se encontró que el tiempo entre el diagnóstico del derrame pericárdico con repercusión hemodinámica y la realización de la ventana pericárdica fue de 2,3 horas.

En los pacientes sin taponamiento cardiaco u otro signo de repercusión hemodinámica, el procedimiento quirúrgico se programa en los siguientes tres días después del diagnóstico del derrame pericárdico. Después de la ventana pericárdica, el $92 \%$ de los pacientes son trasladados a recuperación y vigilados estrechamente en hospitalización general, con atención cuidadosa de los cambios en la monitorización cardiaca.

El síndrome pospericardiectomía, caracterizado por sobredistensión o dilatación cardiaca, con hipocinesia o hipocontractilidad miocárdica, alteraciones en el ritmo y signos de insuficiencia mitral y tricuspídea, se presentó solo en $4 \%$ de los casos y se manejó exitosamente en cuidados intensivos, con soporte hemodinámico, aminas, betabloqueadores y amiodarona para los casos de fibrilación auricular, y cardioversión farmacológica.

La ventana pericárdica es un procedimiento seguro, con mortalidad intraoperatoria de $0 \%$ y mortalidad posoperatoria de 1,2\%. La morbilidad asociada al procedimiento quirúrgico es aceptable $(2,3 \%)$ y en ninguna de las condiciones empeoró el estado general del paciente, previo al procedimiento quirúrgico. El porcentaje de recurrencia de derrame pericárdico después de este procedimiento fue de $2,1 \%$. La supervivencia global aumenta a ocho meses en $72 \%$ de los pacientes.
Se recomienda la vía toracoscópica de abordaje o la toracotomía antero-lateral sobre la ventana pericárdica subxifoidea, pues permite evaluar bajo visión directa el pericardio, tomar biopsias suficientes del pericardio y de la pleura, practicar pericardiectomía amplia en caso de carcinomatosis pericárdica y pericarditis restrictiva, y evacuar el derrame pleural coexistente en $45 \%$ de los casos; además, permite establecer una vía de drenaje pleuropericárdica ${ }^{33,34}$.

\section{Conclusiones}

El manejo multidisciplinario moderno del cáncer, aun en etapas avanzadas y metastásicas, ha transformado el padecimiento oncológico en una enfermedad crónica, sujeta a complicaciones.

El derrame pericárdico maligno es una urgencia oncológica que puede presentarse en neoplasias sólidas y hematológicas. Requiere un manejo costo-efectivo que sea resolutivo, expedito y duradero, sin agregar morbilidad a un paciente ya con deterioro de su estado general. Requiere de un manejo individualizado, distinguiendo el derrame pericárdico asociado a neoplasia maligna, el derrame pericárdico maligno y la carcinomatosis pericárdica.

La ventana pericárdica por toracoscopia asistida por video (VATS) en pacientes seleccionados y la minitoracotomía antero-lateral, es la vía ideal de abordaje en casos de derrame pericárdico maligno, pues, además de permitir la evacuación del derrame pericárdico, la evaluación bajo visión directa del pulmón, la pleura y el pericardio, la resolución del derrame pleural y la liberación del pulmón atrapado en algunos casos, permite tomar biopsias con tejido suficiente para la identificación histopatológica, la inmunohistoquímica y el perfil molecular del tumor malino primario.

\title{
Management of pericardial effusion in cancer patients
}

\begin{abstract}
Introduction: Pericardial effusion is the most frequent cardiac complication in the cancer patient. Lung cancer and breast cancer are the most common solid neoplasms associated with pericardial effusion. Multimodal oncology management has allowed an increase in overall survival and has exposed oncological complications, which require individualized management for these patients.
\end{abstract}


Objective: We present our experience in the management of pericardial effusion, from its physiopathology, adequate classification in: pericardial effusion associated with malignancy, pericardial effusion and pericardial carcinomatosis. Diagnostic and therapeutic approach.

Results: the incidence of pericardial effusion in our institution is $12 \%$. In almost 100 pericardial window procedures, pericardiectomy and installation of a pericardial or pleuropericardial tunnelled catheter, in 11 years postoperative mortality was $1.2 \%$ and pericardial effusion recurrence was $2.1 \%$ compared to $33 \%$ recurrence in patients Led to pericardicentesis.

Conclusion: Malignant pericardial effusion is an oncologic emergency. It requires cost-effective management in terms of being resolute, expeditious and lasting, with no additional morbidity to a patient, who already appears deteriorated in his general condition. The pericardial window by thoracoscopy, VATS in selected patients and the mini anterolateral thoracotomy are the ideal approach for the patient with malignant pericardial effusion.

Key words: Neoplasms; pericardium; pericardial effusion; pericardial window techniques; survival.

\section{Referencias}

1. Klatt EC, Heitz DR. Cardiac metastases. Cancer. 1990;65: 1456-9.

2. Pohjola-Sintonen S, Tötterman KJ, Salmo M, Siltanen P. Late cardiac effects of mediastinal radiotherapy in patients with Hodgkin's disease. Cancer. 1987;60:31-7.

3. Cullinane CA, Paz IB, Smith D, Carter N, Grannis FW Jr. Prognostic factors in the surgical management of pericardial effusion in the patient with concurrent malignancy. Chest. 2004; $125: 1328-34$.

4. Gornik H, Gerhard-Herman M, Beckman JA. Abnormal cytology predicts poor prognosis in cancer patients with pericardial effusion. J Clin Oncol. 2005;23:5211-6.

5. Tsang TS, Seward JB, Barnes ME, Bailey KR, Sinak LJ, Urban $\mathrm{LH}$, et al. Outcomes of primary and secondary treatment of pericardial effusion in patients with malignancy. Mayo Clin Proc. 2000;75:248-53.

6. Ferlay J, Soerjomataram I, Dikshit R, Eser S, Mathers C, Rebelo $\mathrm{M}$, et al. Cancer incidence and mortality worldwide: Sources, methods and major patterns in GLOBOCAN 2012. Int J Cancer. 2014;136:E359-86.

7. Abraham KP, Reddy V, Gattuso P. Neoplasms metastatic to the heart: Review of 3314 consecutive autopsies. Am J Cardiovasc Pathol. 1990;3:195-8.

8. McDonald JM, Meyers BF, Guthrie TJ, Battafarano RJ, Cooper JD, Patterson GA. Comparison of open subxyphoid pericardial drainage with percutaneous catheter drainage for symptomatic pericardial effusion. Ann Thorac Surg. 2003;76:811-5.

9. Kim SH, Kwak MH, Park S, Kim HJ, Lee HS, Kim MS,et al. Clinical characteristics of malignant pericardial effusion associated with recurrence and survival. Cancer Res Treat. 2010;42:210-6.
10. Akhter SA. The heart and pericardium. Thorac Surg Clin. 2011;21:205-17.

11. Kaiser L, Kron IL, Spray TL. Mastery cardiothoracic surgery. Third edition. Philadelphia: Wolters Kluwer; 2013. p. 289-95.

12. Echeverri D, Matta L. Pericarditis tuberculosa. Biomédica. 2014;34:528-34.

13. Gottdiener JS, Appelbaum FR, Ferrans VJ, Deisseroth A, Ziegler J. Cardiotoxicity associated with high-dosecyclophosphamide therapy. Arch Intern Med. 1981;141:758-63.

14. Refaat MM, Katz WE. Neoplastic pericardial effusion. Clin Cardiol. 2011;34:593-8.

15. Shields TW. General thoracic surgery. Seventh edition. Philadelphia: Lippincott Williams \& Wilkins; 2009. p. 885-90.

16. Tsang TS, Enriquez-Sarano M, Freeman WK, Barnes ME, Sinak LJ, Gersh BJ, et al. Consecutive 1127 therapeutic echocardiographicallyguided pericardiocenteses: Clinical profile, practice patterns, and outcomes spanning 21 years. Mayo Clin Proc. 2002;77:429-36.

17. Burazor I, Imazio M. Malignant pericardial effusion. Cardiology. 2013;124:224-32.

18. Petrofsky M. Management of malignant pericardial effusion. J Adv Pract Oncol. 2014;5:281-9.

19. Jama GM. Palliative treatment for symptomatic malignant pericardial effusion. Interact Cardiovasc Thorac Surg. 2014;19:1019-26.

20. Karnofsky D, Burchenal J. The clinical evaluation of chemotherapeutic agents in cancer. In: MacLeod C, editor. Evaluation of chemotherapeutic agents. New York: Columbia University Press; 1949. p. 191-205. 
21. Oken MM, Creech RH, Tormey DC, Horton J, Davis TE, McFadden ET, et al. Toxicity and response criteria of the Eastern Cooperative Oncology Group. Am J Clin Oncol. 1982;5:649-55.

22. Campbell PT. Subxiphoid pericardiotomy in the diagnosis and management of large pericardial effusions associated with malignancy. Chest. 1992;101:938-43.

23. Hankins JR, Saterfield JR. Pericardial window for malignant pericardial effusion. Ann Thorac Surg. 1980;30:465-71.

24. Sugimoto JT, Little AG, Ferguson MK, Borow KM, Vallera $\mathrm{D}$, Staszak VM, et al. Pericardial window: Mechanisms of efficacy. Ann Thorac Surg. 1990;50:442-5.

25. Celik S, Lestuzzi C, Cervesato E, Dequanter D, Piotti P, De Biasio M, et al. Systemic chemotherapy in combination with pericardial window has better outcomes in malignant pericardial effusions. J Thorac Cardiovasc Surg. 2014;148:2288-93.

26. Patel N, Rafique AM, Eshaghian S, Mendoza F, Biner S, Cercek $\mathrm{B}$, et al. Retrospective comparison of outcomes, diagnostic value, and complications of percutaneous prolonged drainage versus surgical pericardiotomy of pericardial effusion associated with malignancy. Am J Cardiol. 2013;112:1235-9.

27. Girardi LN, Ginsberg RJ, Burt ME. Pericardiocentesis and intrapericardial sclerosis: Effective therapy for malignant pericardial effusions. Ann Thorac Surg. 1997;64:1422-8.

28. Wilkes JD, Fidias P, Vaickus L, Perez RP. Malignancy-related pericardial effusion. 127 cases from the Roswell Park Cancer Institute. Cancer. 1995;76:1377-87.
29. Maisch B, Ristic AD, Pankuweit S, Neubauer A, Moll R. Neoplastic pericardial effusion. Efficacy and safety of intrapericardial treatment with cisplatin. Eur Heart J. 2002;23:1625-31.

30. Dequanter D, Lothaire P, Berghmans T, Sculier JP. Severe pericardial effusion in patients with concurrent malignancy: A retrospective analysis of prognostic factors influencing survival. Ann Surg Oncol. 2008;15:3268-71.

31. Lestuzzi C. Neoplastic pericardial disease: Old and current strategies for diagnosis and management. 2010;2:270-9.

32. Apodaca-Cruz A, Villarreal-Garza C, Torres-Ávila B, Torres J, Meneses A, Flores-Estrada D, et al. Effectiveness and prognosis of initial pericardiocentesis in the primary management of malignant pericardial effusion. Interact Cardiovasc Thorac Surg. 2011;11:154-61.

33. Gillen J,Lau C. Permanent indwelling catheters in the magnagementof pleural effusions. Thoracic Surg Clin.2013;23;63-71.

34. Ahmad MM. The pericardical window: Is a video assisted thoracoscopy approach better than surgical approach? Interact Cardiovasc Thorac Surg. 2011;12:175-8.

Correspondencia: Luis Alberto Chinchilla, MD Correo electrónico:

chinchillasurgery@hotmail.com; Ciudad de México 